\title{
Trial of Essiac to Ascertain Its Effect in Women with Breast Cancer (TEA-BC)
}

\author{
SUZANNA M. ZICK, N.D., M.P.H., ${ }^{1}$ ANANDA SEN, Ph.D., ${ }^{2}$ YANG FENG, M.P.H., ${ }^{3}$ \\ JEN GREEN, N.D., ${ }^{4}$ SHADE OLATUNDE, B.Sc., ${ }^{5}$ and HEATHER BOON, B.Sc.Phm., Ph.D. ${ }^{5}$
}

\begin{abstract}
Background: Breast cancer is a major cause of morbidity, mortality, and medical expenditures among women in Canada. Essiac (Resperin ${ }^{\mathrm{TM}}$ Canada Limited, Waterloo, Ontario, Canada), a blend of at least four herbs (burdock root [Arctium lappa], Indian rhubarb [Rheum palmatum], sheep sorrel [Rumex acetosella], and the inner bark of slippery elm [Ulmus fulva or U. rubra]), has become one of the more popular herbal remedies for breastcancer treatment, secondary prevention, improving quality of life, and controlling negative side-effects of conventional breast-cancer treatment.

Objectives: Our primary objective was to determine the difference in health-related quality of life (HRQOL), as assessed by the Functional Assessment of Cancer Therapy Breast Cancer Version, between women who are new Essiac users (since breast cancer diagnosis) and those who have never used Essiac. Secondary endpoints included differences in depression, anxiety, fatigue, rate of adverse events, and prevalence of complications or benefits associated with Essiac during standard breast-cancer treatment. Additionally, we described the pattern of use of Essiac in this cohort of women.

Methods: We performed a retrospective cohort study in 510 women, randomly chosen from the Ontario Cancer Tumour Registry, with a diagnosis of primary breast cancer in 2003.

Results: With the exception changes in a Physical well-being subscale and a relationship with doctor subscale, Essiac did not have a significant effect on HR-QOL or mood states. Even for Physical well-being and relationship with doctor, Essiac seemed to have a negative effect, with Essiac users doing worse than the nonEssiac users. This might be attributed to the fact that the group of users comprised younger women with more advanced stages of breast cancer, and both of these subgroups of patients have been shown to be at a significantly increased risk for negative mood states and/or a decreased sense of well-being. The women were taking low doses (total daily dose $43.6 \pm 30.8 \mathrm{~mL}$ ) of Essiac that corresponded to the label directions found on most Essiac products. Friends were the most common source of information, and most women were taking Essiac to boost their immune systems or increase their chances of survival. Only 2 women reported minor adverse events, whereas numerous women reported beneficial effects of Essiac.
\end{abstract}

Conclusions: Essiac does not appear to improve HR-QOL or mood states. Future studies are needed to determine whether other clinical outcomes, such as cancer reoccurrence, are affected by Essiac.

\footnotetext{
${ }^{1}$ Integrative Medicine, University of Michigan, Ann Arbor, MI.

${ }^{2}$ Center for Statistical Consulting and Research, University of Michigan, Ann Arbor, MI.

${ }^{3}$ Department of Biostatistics, University of Michigan, Ann Arbor, MI.

${ }^{4}$ Orchard Lake, MI.

${ }^{5}$ Leslie Dan Faculty of Pharmacy, University of Toronto, Toronto, Ontario, Canada.
} 


\section{INTRODUCTION}

$\mathbf{T}$ The importance of breast cancer in women as a public health problem is well-recognized worldwide. ${ }^{1,2}$ In Canadian women, breast cancer is the most common neoplasm, with a lifetime risk calculated as 1 in $9 .{ }^{3}$ As a result of mammographic screening, early detection of disease, and improved therapies, the rate of breast cancer-mortality is steadily declining in Canada. ${ }^{3}$ Nonetheless, in 2005 , it is estimated that 21,600 women were diagnosed and 5300 women died of this disease. ${ }^{3}$ Furthermore, Ontario had the highest incidence of breast cancer cases in Canada, with 8200 of the 21,600 new cases in $2005 .^{3}$

Health-related quality of life (HR-QOL) is becoming an increasingly important endpoint for assessing treatment and management in breast cancer. ${ }^{4,5}$ Conventional endpoints for evaluating cancer treatments include disease-free survival time, tumor response, and overall survival. Over time, however, it has become evident that these endpoints alone are not adequate to make informed decisions about treatment options for breast cancer. Measurements of HR-QOL are recognized as comprising a more complete assessment of patients' physical, emotional, mental, and spiritual well-being. 5,6 Additionally, HR-QOL measures may provide prognostic information independent of other parameters such as disease severity, ${ }^{6,7}$ and some scales have been found to be significantly associated with patients' survival. ${ }^{4}$ Furthermore, HR-QOL scales help to measure the effects of the severity as well as the duration of toxic side effects from treatment (e.g., neuropathy) augmenting information received from conventional toxicity scales. ${ }^{6}$

There have been numerous recent surveys of complementary and alternative medicine (CAM) use among patients with breast cancer in the United States, Britain, and Canada. ${ }^{8-13}$ Survey results among patients with breast cancer find that prevalence of CAM use ranges from $16.5 \%$ to $84 \% .{ }^{8-13}$ In a study examining the prevalence of CAM use among Ontario women with breast cancer, $67 \%$ of the women surveyed used some form of alternative therapies. Of these women, almost $15 \%$ used Essiac (Resperin ${ }^{\mathrm{TM}}$ Canada Limited, Waterloo, Ontario, Canada), an herbal remedy, which was the second most commonly used natural health product after green tea (Camellia sinensis) $(17.3 \%){ }^{8}$ Moreover, in the United States, a survey of Flor-Essence ${ }^{\circledR}$ (an Essiac formula, Flora Manufacturing and Distributing, Burnaby, British Columbia, Canada) users found that, of 5051 respondents, $22 \%$ had breast cancer. ${ }^{14,15}$ An estimate of annual U.S. Essiac sales exceeded $\$ 8$ million in 2001. ${ }^{14}$ An international study conducted by the Canadian Cancer Society's Cancer Information Services (CIS) to identify the information needs of those who called CIS requesting information on CAM found that, of the 109 callers, the majority were women $(80 \%)$, most of their questions concerned the safety and/or effectiveness of natural health products, and breast cancer was the most common type of cancer queried $(22 \%)$. Of these queries, the top CAM topics most often asked about were $714 \mathrm{X}(N=17)$ and $\operatorname{Essiac}(N=16)$. The study author concluded that more cancer-specific information on Essiac use in breast cancer is needed. ${ }^{16}$

Essiac has been used in Canada for more than 70 years. ${ }^{17}$ The recipe for Essiac is said to have been given to a nurse, Rene Caisse, by 1 of her patients who reportedly learned of the mixture from an Ojibwa healer and claimed it cured her breast cancer. Beginning in the 1920s, Caisse administered Essiac to hundreds of patients with cancer for more than 40 years. $^{17}$

The original Essiac recipe contained four herbs: burdock root (Arctium lappa); Indian rhubarb (Rheum palmatum); sheep sorrel (Rumex acetosella); and the inner bark of slippery elm (Ulmus fulva or U. rubra). Caisse administered one of the herbs by injection and the others as a tea. By 1938 , however, concerns about the use of Essiac led to an investigation by the Cancer Commission. ${ }^{17}$ Evidence of 1 report of death and 1 report of toxicity after Essiac tea injections ${ }^{18,19}$ led to the practice of injecting Essiac herbs being deemed unsafe because of a potential for systemic toxicity. Between 1959 and 1978, Caisse worked in collaboration with an American physician, Charles Brusch, M.D., adding four additional herbs to the original recipe: watercress (Nasturtium officinale); blessed thistle (Cnicus benedictus); red clover (Trifolium pretense); and kelp (Laminaria digitata). This new mixture did not require injection and could thus be used at home. Essiac became available to consumers in both the 4- and 8-herb varieties in the 1990s. Today, more than 40 different Essiac formulas are available throughout North America, Australia, and the United Kingdom. ${ }^{14}$

Despite the widespread use of and interest in Essiac among women with breast cancer, there are no published clinical trials and very little animal or in vitro work, most of which is also unpublished. Available Essiac research is difficult to assess because of a lack of information about methodology, differences in Essiac formulations studied, and variance in the experimental animal models in in vivo studies. Anecdotal reports indicate that Essiac may be of benefit in the treatment of cancer, but such reports are limited by their subjective and qualitative nature. Much of the research conducted is thus restricted by poor design, small sample size, lack of control, unclear product stability and/or consistency, and lack of physician cooperation. Given the limitations to date, a well-designed, population-based study on the effects of Essiac is warranted.

The primary objective of our study was to determine the difference in HR-QOL as assessed by the Functional Assessment of Cancer Therapy Breast Cancer Version (FACTB) between women who are new Essiac users since breast cancer diagnosis and those who have never used Essiac. Secondarily, the study was designed to determine the effect of Essiac in women with a primary diagnosis of breast cancer who are new Essiac users since breast cancer diagnosis compared to women who have never used Essiac on the following secondary endpoints: 
1. Depression, anxiety, and fatigue as measured by the Profile of Mood Syndromes (POMS)

2. Prevalence of complications during standard breast cancer treatment (chemotherapy radiation, surgery, and hormonal therapy) as determined by self-report

3. Prevalence of any adverse events (e.g., rash, stomach upset) occurring after standard therapy as determined by self-report.

The third objective was to describe Essiac use in women with a primary diagnosis of breast cancer including:

1. Brand used

2. Duration and continuity of use (e.g., used for 3 years but stopped for 1 month during treatment)

3. Frequency and schedule of use (e.g., 1 cup, three times per day)

4. Method of preparation (if applicable)

5. When use was initiated and stopped

6. Adverse effects associated with use.

\section{MATERIALS AND METHODS}

This was a retrospective study design examining the effect of Essiac on women with breast cancer. Women who were 18 years or older, had been diagnosed with primary breast cancer (positive pathology report) between 2001 and 2003, and able to read and write in English, were eligible to participate in the study. Any woman who had been diagnosed with any type of cancer prior to a diagnosis of breast cancer or who had previously used Essiac was excluded from the study.

The sample was a computer-generated random selection from the Ontario Cancer Registry of those women diagnosed with primary breast cancer in $2003(N=2541)$, subject to the requirements and protocols of the Registry. We attempted to obtain permission to contact each patient and confirmation of current mailing address from her oncologist following the Registry's protocol. Oncologists who did not respond to a letter requesting permission to contact their patients received up to three follow-up telephone calls. In total, we received responses regarding 1453 patients $(57.2 \%)$ and per-

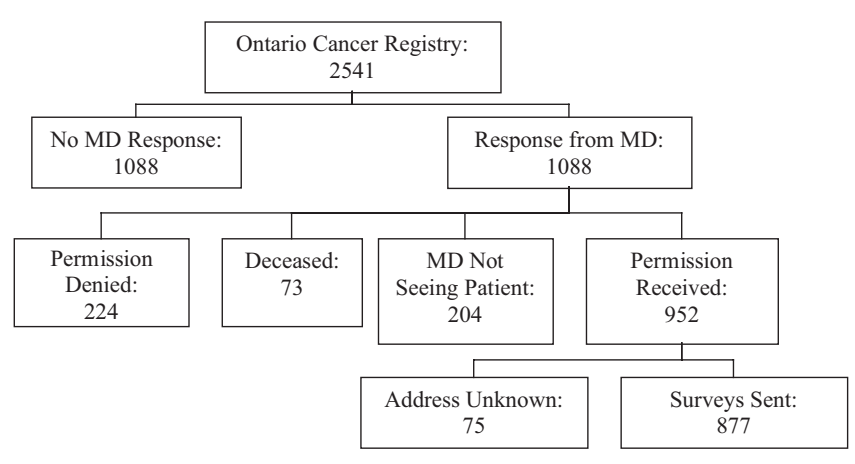

FIG. 1. Derivation of the sample. mission to contact 952 patients $(37.5 \%)$; however, mailing addresses were only available for 877 (34.5\%) (Fig. 1).

A modified Dillman ${ }^{20}$ protocol was used to survey the random sample of patients with breast cancer selected from the Ontario Cancer Registry. A cover letter, questionnaire, and stamped, self-addressed return envelope were mailed to each woman in the entire sample $(N=877)$. Failure to respond to the original and two follow-up mailings was considered a refusal to participate in the study.

\section{The survey instruments}

Each woman received a packet that contained 5 surveys including (1) the FACT-B, ${ }^{21}$ which is a 44 -item self-administered, 5-point Likert scale for evaluating functional impairment and the perceived effect of that impairment on quality of life; (2) POMS, ${ }^{22}$ which is a measure of patient distress; (3) Yale Social Support Index (YALE), ${ }^{23}$ which is used to assess the amount and quality of social contact and support; (4) Patterns and Use of Essiac, which measures whether a study participant has used Essiac, which brand, timeline, amount, and adverse effects; and (5) Prevalence of Use of Complementary/Alternative Therapies, which is based on a survey developed by Yates et al. ${ }^{24}$ and modified to be relevant to Canadians. ${ }^{25}$ At the end of the prevalence survey, participants were also asked about any conventional medical treatments for their breast cancer, stage of disease at diagnosis, current disease state, and demographic data.

\section{Statistical analysis}

Clinical and sociodemographic characteristics of Essiac users and non-Essiac users were compared using an independent samples $t$-test and a chi-square test, for continuous and categoric variables, respectively. For categoric measurements, the chi-square test was replaced by Fisher's exact test, whenever the observed percentage in any crossclassified category fell below 10. Frequencies, percentages, and means were calculated to describe the pattern of Essiac use, who recommended Essiac, and reasons for Essiac use.

Summary score for each subscale of FACT-B and POMS was constructed by first computing an itemwise average by dividing the total score by the number of items responded and then multiplying this average by the total number of items in the subscale. The scores for individuals who responded to less than $75 \%$ of the items were considered to be missing. Also, although each subscale has both positively and negatively framed items, for ease of comparison and interpretation, we reverse coded all positively framed questions, so that a higher average score for any scale signified a worse state.

For continuous variables (FACT-B, POMS), initial exploratory analysis was carried out using independent samples t-tests comparing women who used Essiac to nonusers. Subsequently, the subscales of FACT-B and POMS were used as continuous outcomes in a multiple-regression framework. For these models, Essiac use (yes/no) was entered into the equation as an independent variable simultaneously 
along with age, marital status, income, educational status, stage of disease, months since diagnosis, and number of individuals in self-reported social network as reported by the YALE questionnaire to determine their effects on mood states and HR-QOL. The variables entered into the regression models were chosen primarily because of the general consensus among researchers in this area about their potential effects on mood states or HR-QOL outcomes. In all the multiple regression models with FACT-B and POMS subscales as outcomes, the residuals demonstrated substantial departure from normality, thereby rendering the linear model an improper fit. We conducted systematic analyses with transformed models using Box-Cox family of power transformations, ${ }^{26}$ and found that a square-root transformation of the outcomes worked well in linearizing the relationship. All the regression results we present in this article are thus for the square-root transformed models.

We found it difficult to treat the FACT-B subscale "relationship with doctor" as a continuous outcome. This was primarily because the subscale is based only on two questions, with many respondents answering 0 to both questions $(>50 \%)$. Thus we treated this single variable in a logistic regression framework (score $=0$ or positive) with the same predictors as described above.

Data were input into SPSS Windows version 12 (SPSS, Chicago, IL), then manipulated and analyzed using both SPSS and SAS version 9.1. (SAS Institute Inc, Cary NC). A $p$-value of less than 0.05 was considered to be statistically significant.

The study was designed to have $80 \%$ power to detect a treatment difference of 7 points in FACT-G, based on published standard deviations of 13.9-15.9 in similar populations with a mean of 67-91, ${ }^{27}$ assuming a two-sided $\alpha$ level of 0.05 . This design required $n=120$ per treatment group.

\section{RESULTS}

A total of 541 of surveys were returned. Sixteen (16) families contacted us to inform us that the respondents had died, and 57 envelopes were returned marked "address unknown." Thus, our final response rate was $63 \%(541 /(877-16))$. Of the 541 surveys, 31 were deemed to be ineligible, primarily because the respondents' dates of diagnosis were before 2003, leaving 510 eligible participants. A total of 41 respondents (8\%) reported using Essiac, of which 32 (6.2\%) reported using Essiac for treating their breast cancer.

\section{Sociodemographic and clinical characteristics}

We present the sociodemographic and clinical characteristics of women in the study by Essiac use in Table 1. Women who used Essiac were significantly younger (55 versus $62 ; p=0.005)$ and more likely to be married $(p=0.04)$. In addition, Essiac users were diagnosed with breast cancer at a later stage $(p=0.03)$ and were more likely to have received chemotherapy $(p=0.001)$. There were no significant differences in ethnicity, educational level, total annual household income, rates of estrogen or progesterone positive cancers, or other-breast cancer treatment options (e.g., surgery) between women who used Essiac and nonusers.

\section{Reasons and patterns of Essiac}

Women listed a variety of reasons for using Essiac. The three most common reasons for taking Essiac were "to boost my immune system" $(N=22 / 73 \%)$, "to improve my chances of survival" $(N=22 / 73 \%)$, and "to prevent a recurrence of cancer" $(N=19 / 63 \%)$. Most women had a friend who recommended Essiac to them $(N=13 / 42 \%)$. Other women self-referred $(N=6 / 19 \%)$, had family members $(N=5 / 16 \%)$, or naturopathic physicians $(N=6 / 19 \%)$ recommend Essiac to them. Only 1 woman decided to take Essiac from information obtained from the media.

Two thirds of the women using Essiac for breast cancer purchased an Essiac product. Although there are more than 40 brands available commercially in Ontario, the majority of women purchased the Essiac $(N=18 / 75 \%)$ brand, which contains the 4-herb blend, with only a few women purchasing Flor-Essence $(N=4 / 17 \%)$, which contains the 8-herb blend, or other miscellaneous brands $(N=3 / 13 \%)$. A quarter of the women bought multiple brands of Essiac products $(N=6 / 26 \%)$. A third of women either made the Essiac tea themselves $(N=1 / 3 \%)$ or had someone else make it for them $(N=9 / 31 \%)$ (e.g., a family member or nurse).

The majority of women took Essiac either once or twice per day $(N=26 / 93 \%)$, with a very small minority taking it three times per day $(N=2 / 7 \%)$. The average daily dose of Essiac was $43.6( \pm 30.8)$ and ranged from 12 to $114 \mathrm{~mL}$ per day. The mean length of Essiac use was 11.1 ( \pm 8.9) months, but ranged anywhere from 1 to 28 months.

\section{Adverse events and interactions with breast cancer treatment}

Only two women reported having adverse effect from taking Essiac, and both of these were complaints of nausea and an unpleasant aftertaste. No women reported that Essiac interfered with their breast-cancer treatment. In contrast, 6 women reported experiencing positive interactions between Essiac and their treatment for breast cancer. Positive interactions reported included a decrease in negative side-effects, increased effectiveness, decreased fatigue, and diminished nausea and vomiting from breast cancer treatment. In addition, women also perceived that Essiac allowed them to take their treatments in a timely fashion and that Essiac supported their immune systems during treatments.

\section{Quality of life and mood states}

We found that Essiac users and nonusers were significantly different with respect to the FACT-B physical well-being 
Table 1. Sociodemographics and Clinical Characteristics

\begin{tabular}{|c|c|c|}
\hline Characteristics & $\begin{array}{c}\text { Essiac nonusers } \\
\quad(\mathrm{N}=469)^{\mathrm{a}}\end{array}$ & $\begin{array}{c}\text { Essiac users } \\
(\mathrm{N}=41)^{\mathrm{a}}\end{array}$ \\
\hline Age, mean (STD), years ${ }^{\mathrm{b}}$ & $61.6(13.3)$ & $55.4(12.7)$ \\
\hline \multicolumn{3}{|l|}{ Ethnicity, no. (\%) } \\
\hline North American & $295(63)$ & $22(54)$ \\
\hline European & $74(16)$ & $7(17)$ \\
\hline Multiethnic & $37(8)$ & $5(12)$ \\
\hline Otherc & $35(7)$ & $4(10)$ \\
\hline \multicolumn{3}{|l|}{ Education, no. (\%) } \\
\hline Completed high school & $185(39)$ & $17(41)$ \\
\hline Completed college/university degree & $184(39)$ & $19(46)$ \\
\hline Completed grade 8 & $67(14)$ & $1(2)$ \\
\hline Less than grade 8 & $17(4)$ & $2(5)$ \\
\hline \multicolumn{3}{|l|}{ Income ${ }^{\mathrm{d}}$ no. $(\%)$} \\
\hline$<\$ 20,000$ & $83(18)$ & $7(17)$ \\
\hline$\$ 20,000-\$ 30,999$ & $83(18)$ & $5(12)$ \\
\hline$\$ 40,000-\$ 59,999$ & $84(18)$ & $5(12)$ \\
\hline$\$ 60,000-\$ 79,999$ & $54(11)$ & $4(10)$ \\
\hline$\$ 80,000$ or more & $85(18)$ & $13(32)$ \\
\hline \multicolumn{3}{|l|}{ Marital status, no. $(\%)^{\mathrm{e}}$} \\
\hline Married/common-law & $295(63)$ & $32(78)$ \\
\hline Widowed & $83(18)$ & $1(2)$ \\
\hline Divorced/separated & $59(13)$ & $5(12)$ \\
\hline Never married & $24(5)$ & $2(5)$ \\
\hline \multicolumn{3}{|l|}{ Stage at diagnosis, no. $(\%)^{\mathrm{e}}$} \\
\hline Stage I & $207(44)$ & $12(29)$ \\
\hline Stage II & $109(23)$ & $15(37)$ \\
\hline Stage III & $57(12)$ & $6(15)$ \\
\hline Stage IV & $5(1)$ & $2(5)$ \\
\hline \multicolumn{3}{|l|}{ Treatments for breast cancer, no. (\%) } \\
\hline Surgery & $429(91)$ & $36(88)$ \\
\hline Radiation & $312(67)$ & $31(76)$ \\
\hline Tamoxifen & $245(52)$ & $19(46)$ \\
\hline Chemotherapy ${ }^{\mathrm{b}}$ & $198(42)$ & $28(68)$ \\
\hline \multicolumn{3}{|l|}{ Breast cancer receptor status, no. (\%) } \\
\hline Estrogen positive & $217(46)$ & $23(56)$ \\
\hline Progesterone positive & $57(12)$ & $6(15)$ \\
\hline Months since diagnosis, mean (SD) & $27.3(4.65)$ & $27.6(5.41)$ \\
\hline
\end{tabular}

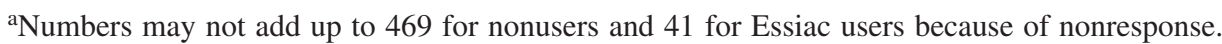

${ }^{\mathrm{b}} p$ value $<0.01$.

'South Asian, Filipino, Arab/Middle Eastern, Chinese, Japanese, African, Caribbean, Aboriginal, Latin American, Unknown.

${ }^{\mathrm{d}}$ Total household income in Canadian dollars.

e $p$ value $<0.05$.

STD, standard deviation.

scale, both in the unadjusted analysis (Table $2 ; p$ value $=$ 0.02 ) and in the adjusted analysis (Table $2 ; p$ value $=0.02$ ) Women who used Essiac reported worse physical well-being than the nonusers. We obtained a similar result for the relationship with doctor subscale. In a logistic regression analysis, the Essiac user group was found to be marginally worse with respect to patient-doctor relationship than the nonusers with an estimated odds ratio of 0.40 ( $p$-value $=0.047,95 \%$ C.I. for odds ratio: [0.16, 0.988]). For all other FACT-B subscales, no statistically significant difference was obtained between the users and nonusers. In all cases, however, women who used Essiac consistently had higher scores, indicating more severe symptoms than nonusers.
Table 3 indicates the association of other potential confounders with FACT-B. Age turned out to have significant associations with physical and emotional well-being, with older women appearing to cope better than the younger ones. Stage of cancer also had significant associations with these two outcomes, with patients diagnosed with more advanced stage faring worse. Income level has a significant association with social well-being only. For all subscales, however, the women in the lower-income groups scored higher (worse) on average. The variable "number of supports" had the strongest association with the FACT-B scale because it was negatively associated with all the subscales, indicating a large impact of social support on the HR-QOL. Education, 
Table 2. Results of Unadjusted and Adjusted Analyses for FACT-B

\begin{tabular}{|c|c|c|c|c|}
\hline Subscales & $\begin{array}{c}\text { Nonusers } \\
\text { mean (STD) }\end{array}$ & $\begin{array}{l}\text { Essiac users } \\
\text { mean (STD) }\end{array}$ & $\mathrm{p}$ value ${ }^{* 1}$ & Adjusted $\mathrm{p}$ value ${ }^{* 3}$ \\
\hline Physical well-being & $4.0(4.3)$ & $5.7(5.0)$ & 0.02 & 0.02 \\
\hline Social well-being & $4.5(4.7)$ & $4.5(4.7)$ & 0.85 & 0.88 \\
\hline Emotional well-being & $4.5(4.1)$ & $5.4(4.4)$ & 0.18 & 0.23 \\
\hline Functional well-being & $6.4(5.4)$ & $6.9(6.3)$ & 0.57 & 0.83 \\
\hline Relationship with doctor & $1.3(1.7)$ & $1.5(1.7)$ & $0.35 * 2$ & 0.05 \\
\hline
\end{tabular}

* $p$ values (1) are based on independent sample $t$-test comparing the mean of Essiac users to nonusers except for relationship with doctor, which is (2) presented as Fisher's Exact test comparing scores of 1 versus 0; (3) adjusted analyses are based on multiple linear regressions with the well-being subscales as outcomes, adjusted for age, marital status, income, educational status, stage of disease, months since breast cancer diagnosis and number of individuals in self-reported social network as reported by the Yale Social Support Index. For relationship with doctor, the adjusted analysis is based on logistic regression.

FACT-B, Functional Assessment of Cancer Therapy Breast Cancer Version; STD, standard deviation.

marital status, and months since diagnosis had no significant association with any of the subscales.

We also found no significant difference in either unadjusted or adjusted analyses for any of the six subscales of the POMS between women who used Essiac and women who had never used Essiac (Table 4). In general, Essiac users also had higher mean scores on four of the POMS subscales indicating more "mood" disturbances, such as anxiety. Table 5 shows the effect of various covariates on POMS. Of these, age seemed to have a significant association with most of the subscales considered. For example, older women appeared to be significantly less anxious, depressed, confused, angry, and fatigued compared to the younger ones on average. As for the FACT-B QOL scales, number of people in women's support network also has a large positive impact on the mood states. Both education level and stage of disease have mild impact on the mood states, each being significantly associated with one of the scales. On the other hand, income level seems to have a modest impact, with women at a lower income category generally at a worse state of mood than their wealthier counterparts. Once again, similar to the FACT-B QOL measures, marital status and months since diagnosis had no association with the mood states.

\section{DISCUSSION}

We found that $8.1 \%$ of Ontario women with breast cancer take Essiac and, of these women, approximately $75 \%$ use Essiac for breast cancer. It is unknown why the $25 \%$ of women who were not using Essiac specifically for their breast cancer were using the product. The overall percentage of Essiac users is similar (8.1\% vs. $9 \%$ ) to a previous survey conducted by Boon ${ }^{8}$ in a cohort of women drawn from the Ontario Cancer Registry and diagnosed with breast cancer in 1994 or 1995. In addition, the majority of women using Essiac in our study bought either Essiac or FlorEssence brands and appeared to be taking doses recommended on Essiac or Flor-Essence labels.

Our study indicated that the doses, frequencies, and brands of Essiac used by the women in this study did not have any effect on either disease-specific quality of life, as measured by the FACT-B, or various mood states including

Table 3. Summary of Regression Results of FACT-B Subscales

\begin{tabular}{|c|c|c|c|c|c|}
\hline Subscales & Essiac use & Age & $\begin{array}{l}\text { Stage at } \\
\text { diagnosis }\end{array}$ & $\begin{array}{c}\text { Number of } \\
\text { supports }\end{array}$ & Income \\
\hline Physical well-beingal & $0.50(0.22) *$ & $-0.02(0.01)^{* *}$ & $0.18(0.08)^{*}$ & $-0.02(0.01)^{*}$ & $-0.10(0.05)$ \\
\hline Social well-beingal & $0.04(0.26)$ & $-0.00(0.01)$ & $-0.07(0.09)$ & $-0.05(0.01)^{* *}$ & $-0.15(0.06)^{*}$ \\
\hline Emotional well-beinga1 & $0.25(0.21)$ & $-0.02(0.01)^{* *}$ & $0.21(0.08)^{* *}$ & $-0.02(0.01)^{*}$ & $-0.03(0.05)$ \\
\hline Functional well-beinga1 & $0.17(0.24)$ & $-0.00(0.01)$ & $0.11(0.09)$ & $-0.02(0.01)^{*}$ & $-0.11(0.06)$ \\
\hline Relationship with doctor ${ }^{\mathrm{a} 2}$ & $0.40(0.18)^{*}$ & $1.00(0.01)$ & $1.02(0.15)$ & $0.96(0.02)^{*}$ & $1.05(0.11)$ \\
\hline
\end{tabular}

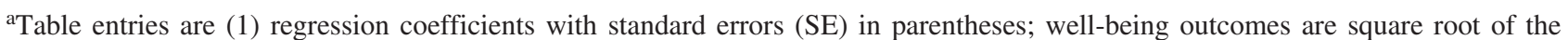
subscale scores obtained from the survey for the well-being subscales, (2) and Odds Ratio (SE) for relationship with doctor, relationship with doctor is a dichotomous outcome; all regression models are additionally adjusted for marriage status (currently married or not), education level, and months since diagnosis. Negative regression coefficients indicate that the lower the value (e.g., lower age, income) the worse the woman's well-being; the opposite relationship is also true (e.g., lower stage/severity at time of diagnosis the better the woman's well-being).

$* p$-value $<0.05$.

$* * p$-value $<0.01$. 
Table 4. Results of Unadjusted and Adjusted Analyses for Profile of Mood States

\begin{tabular}{|c|c|c|c|c|}
\hline Subscales & $\begin{array}{c}\text { Nonusers } \\
\text { mean (STD) }\end{array}$ & $\begin{array}{l}\text { Essiac users } \\
\text { mean (STD) }\end{array}$ & $\mathrm{p}$ value ${ }^{* 1}$ & Adjusted $\mathrm{p}$ value ${ }^{* 2}$ \\
\hline Anxiety & $3.9(3.8)$ & $4.4(4.2)$ & 0.46 & 0.41 \\
\hline Confusion & $4.5(2.7)$ & $4.9(3.1)$ & 0.39 & 0.57 \\
\hline Depression & $3.3(3.8)$ & $3.1(4.0)$ & 0.72 & 0.86 \\
\hline Anger & $2.9(3.4)$ & $2.9(4.0)$ & 0.90 & 0.72 \\
\hline Fatigue & $6.3(7.3)$ & $7.3(5.1)$ & 0.23 & 0.11 \\
\hline Vigor & $11.5(4.9)$ & $11.6(5.4)$ & 0.83 & 0.51 \\
\hline
\end{tabular}

* $p$ values (1) are based on independent sample $t$-test comparing the mean of Essiac users to nonusers and (2) adjusted analyses are based on multiple linear regressions with the mood subscales as outcomes, adjusted for age, marital status, income, educational status, stage of disease, months since breast cancer diagnosis, and number of individuals in self-reported social network as reported by the Yale Social Support Index.

STD, standard deviation.

depression and anxiety. This was despite the finding that Essiac users were significantly worse than regarding physical well-being compared to nonusers. We propose that Essiac use is just a marker of physical distress and not its cause. Instead our data appear to indicate that younger women with more advanced disease and lacking in a sufficient social support network are more likely to have the worst physical well-being and, thus, more likely to try unconventional therapies. These findings are not dissimilar to other surveys in women with breast cancer in which women with more severe disease $\mathrm{e}^{13,28-30}$ and younger age $\mathrm{e}^{8,9,31-34}$ used more CAM therapies. These results are in contrast to those of an unpublished 1977 report of a Phase II clinical study that determined that, although Essiac did not offer clinical benefit to patients with cancer in terms of either survival or tumor regression, there were significant subjective improvements in symptom control and well-being. ${ }^{17,18,{ }^{*}}$ Also, in 1997, an abstract based on the Meeting Program Proceedings of the

*Caisse R. Essiac: A treatment for cancer: Report of patients presented to The Royal Cancer Commission of Ontario (Canada). Unpublished report; 1938.
American Society of Clinical Oncology identifying 59 patients who had taken Essiac in a retrospective review of 360 patients with breast, prostate, or gastrointestinal (GI) cancer. According to the subjective reports of these patients, $30 \%$ of patients on Essiac believed that it helped their quality of life. ${ }^{18}$ There are several possible explanations for the differences in our study findings. First, the women in our study may have been taking doses that were much lower (around $1 / 4$ cup per tea daily) than in other studies, and these low doses may have been insufficient to produce a physiologic effect. Second, the manner in which quality of life was assessed between the studies was different, with both of the other studies using self-reports and not validated quality-oflife scales.

In our study, Essiac's lack of effect on quality of life and mood states may be a true finding, but Essiac could have positive effects on tumor growth and reoccurrence measures that our study was not designed to detect. There is limited evidence from animal and in vitro studies showing antiproliferative effects of Essiac in cancer cell lines, although most of the evidence shows no effect. A study of 8 mice injected with human carcinoma cells revealed apparent tumor de-

Table 5. Summary of Regression Results of Profile of Mood States (POMS) Subscales ${ }^{\mathrm{a}}$

\begin{tabular}{lcccccc}
\hline Subscales & Essiac use & Age & Income & $\begin{array}{c}\text { Stage at } \\
\text { diagnosis }\end{array}$ & $\begin{array}{c}\text { Number of } \\
\text { supports }\end{array}$ & \multicolumn{1}{c}{ Education } \\
\hline Anxiety & $0.16(0.22)$ & $-0.02(0.01)^{*}$ & $-0.09(0.05)$ & $0.02(0.08)$ & $-0.02(0.01)^{*}$ & $-0.12(0.08)$ \\
Confusion & $0.07(0.13)$ & $-0.02(0.00)^{*}$ & $-0.07(0.03)^{* *}$ & $0.03(0.05)$ & $-0.00(0.00)$ & $0.00(0.05)$ \\
Depression & $-0.04(0.22)$ & $-0.03(0.01)^{*}$ & $-0.14(0.05)^{* *}$ & $0.16(0.08)^{* *}$ & $-0.03(0.01)^{*}$ & $-0.07(0.08)$ \\
Anger & $-0.07(0.21)$ & $-0.03(0.01)^{*}$ & $-0.11(0.05)^{* *}$ & $0.02(0.08)$ & $-0.01(0.01)^{* *}$ & $-0.13(0.08)$ \\
Fatigue & $0.37(0.23)$ & $-0.02(0.01)^{*}$ & $-0.13(0.06)^{* *}$ & $0.06(0.08)$ & $-0.02(0.01)^{*}$ & $-0.07(0.09)$ \\
Vigor & $0.11(0.17)$ & $0.01(0.01)$ & $-0.05(0.04)$ & $0.02(0.06)$ & $-0.02(0.01)^{*}$ & $-0.16(0.06)^{* *}$ \\
\hline
\end{tabular}

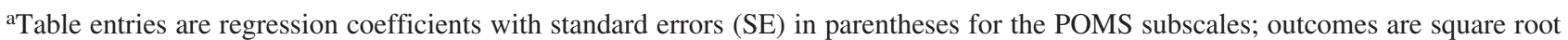
of the subscale scores obtained from the survey for the subscales scores; all regression models are additionally adjusted for marriage status (currently married or not), education level, and months since diagnosis. Negative regression coefficients indicate that the lower the value (e.g., lower age, income) the worse the woman's mood state; the opposite relationship is also true (e.g., lower stage/severity at time of diagnosis the better the woman's mood state).

$* p$ value $<0.01$.

$* * p$ value $<0.05$ 
generation after receiving Essiac. However, all observations confirming tumor necrosis and cellular degradation in the mice were narrative and not documented in terms of histologic parameters. ${ }^{35}$ A recent in vitro study using Essiac and Flor-Essence teas on human tumor cell lines (MCF7, MDAMB-468, Jurkat, and HL60 cells) demonstrated that both Essiac and Flor-Essence herbal teas demonstrated antiproliferative and differentiation-inducing properties in vitro only at high concentrations (1/10 and 1/100 dilutions) that are unlikely to be obtained at doses being consumed by humans. ${ }^{36}$ In contrast, 6 immunotherapy and 2 chemotherapy tests on 2 dried and 1 liquid sample of Essiac provided by Ms. Caisse to the Memorial Sloan-Kettering Cancer Center, New York, NY (MSKCC) showed no in vivo activity. ${ }^{37}$ Researchers admit, however, that the negative results might have been caused by the use of an inappropriate animal system and difficulties encountered in the preparation, administration, and stability of Essiac. ${ }^{37}$ Furthermore, in another set of 17 studies conducted by MSKCC testing Resperin, ${ }^{\mathrm{TM}}$ a four-herb Essiac blend, in animal leukemia and solid tumor test systems showed no antitumor activity. ${ }^{37}$ In a similar fashion, the National Cancer Institute tested a liquid Essiac sample in 1983 and found no antitumor activity in the mouse lymphocytic leukemia P388 tumor system. ${ }^{37}$

With more encouraging results, several human studies have noted an improvement in tumor growth after individuals took Essiac tea. In a study conducted by an Israeli marketer, 20\% showed tumor regression and 5\% healed completely. This study, however, was not subjected to peer-review. ${ }^{38}$ In a 1938 unpublished report, Caisse documented the progress of 24 cancer patients taking Essiac. All patients experienced either complete or partial "cure," but it is unclear how these outcomes were measured. ${ }^{\dagger}$ In 1939, 49 of Caisse's patients gave testimonials to the Canadian Cancer Commission on Caisse's behalf. Of the eight patients whose diagnoses were confirmed, the commission concluded that only two of the four recoveries could be attributed to Essiac. ${ }^{39}$ In contrast, a Phase II clinical study determined that Essiac did not offer clinical benefit to cancer patients in terms of either survival or tumor regression. ${ }^{17,18, *}$ In the early 1980s, the Canadian Department of National Health and Welfare reviewed data from 86 patient histories submitted from 74 of 150 physicians supervising the care of patients on Essiac between 1978 and $1982 .{ }^{40}$ Essiac was shown to have no benefit in 47 patients, 17 patients died, 8 were not evaluable based on the information provided, 1 demonstrated subjective improvement, 5 required fewer analgesics, 4 had objective responses, and 4 remained stable with respect to disease progression. ${ }^{41,42}$ Unfortunately, because the majority of these studies are unpublished, it is

$\dagger$ Flora Manufacturing and Distributing Files (Unpublished Data): Academy of Agricultural Sciences of the Russian Federation Research \& Manufacturing Association Research \& Development Institute of Medical \& Aromatic Plants; 1996. difficult to assess the true effect of Essiac on tumor growth and recurrence.

The women who used Essiac in our study are similar to patients with breast cancer who have used CAM overall in terms of demographic and clinical characteristics. Women who used Essiac were significantly younger, were diagnosed with breast cancer at a later stage, and are more likely to receive chemotherapy. Many other studies in patients with breast cancer have found that younger age is associated with higher prevalence of CAM use, ${ }^{8,9,31-34}$ and several other surveys have found significant correlations between CAM use and adjuvant chemotherapy, ${ }^{8,29,31,32,34}$ and stage of diagnosis, ${ }^{13,28-30}$ and presence of recurrence or metastasis of cancer. ${ }^{32,34}$ Women who were taking Essiac did differ from overall CAM users in terms of educational attainment. In contrast to other surveys in women with breast cancer, $, 8,12,29,32-34,43$ in which women with higher levels of education were more likely to use CAM, we did not observe any significant difference in education between Essiac users and nonusers.

Women who were taking Essiac also stated reasons that were similar to those of women using other CAM therapies for breast cancer. Most women in various surveys, including ours, stated that they used CAM for some reason related to helping their bodies to fight cancer or to boost their immune systems, ${ }^{8,11,30,43}$ as well as to help improve their chance of survival or to decrease the chance of a recurrence of their cancer and restore hope for the future. ${ }^{8,11,44}$ Essiac users also tend to get their information about Essiac from the same sources as women with breast cancer get their information about other types of CAM: from friends and family members. ${ }^{9,30}$

Women in our study reported only two adverse events involving mild GI upset and an unpleasant taste. No women reported any negative interactions with her conventional breast-cancer treatment, whereas several women reported positive benefits including decreased side-effects during radiotherapy and chemotherapy that they attributed to taking Essiac concurrently with their conventional treatments. The lack of adverse events and negative interactions with adjuvant cancer treatments in our study is confirmed by existing reports in the literature concerning Essiac lack of toxicity. Ever since evidence of 1 report of death and one report of toxicity after Essiac tea injections ${ }^{18,19}$ in the 1930s, the practice of injecting Essiac herbs has been considered unsafe because of a potential for systemic toxicity. Instead, Ms. Caisse introduced the blend of herbs used today, orally. Preclinical and clinical evaluations of Flor-Essence, one of the most popular brands of Essiac tea, have assessed acute and chronic toxicity, determining no lethal dose level in acute toxicity studies with albino mice and rats, nor any renal or hepatic toxicity doses in chronic toxicity tests with albino mice, rats (doses were 10 times greater than therapeutic dose at $15 \mathrm{~mL} / \mathrm{kg}$ ), and dogs (doses were five times greater than therapeutic dose at $7.5 \mathrm{~mL} / \mathrm{kg})^{39}$ Several stud- 
ies in humans have also reported no or very few adverse events. ${ }^{18,38}$ When adverse events are reported, they are limited to minor cases of GI upset and unpleasant taste. Consequently, it appears that Essiac is safe and relatively tolerable in the doses recommended and consumed in our study.

Several limitations of our study need to be addressed. As with any retrospective study, recall bias is a possible limitation. However, the women in this study were asked to recall no more than 2 years into the past and some as little as 1 year. Consequently, we posit that only very detailed information such as the exact dose of Essiac would tend to be inaccurately remembered. Another limitation of this study was the low overall response rate and thus a low number of Essiac users. The low response rate compared to a previous survey in a similar population ${ }^{8}$ was a result of sending out the survey in summer, when many women were away on holiday, and the establishment of new privacy rules to protect patients. In addition, a switch from paper to electronic records by the Ontario Cancer registry made it very difficult to contact potential study participant's primary care practitioners or oncologists and in association with the privacy laws, made clinic staff reluctant to release patient information.

Despite our low number of Essiac users, we were still able to determine meaningful demographic and clinical differences in these women, as well as establishing patterns of Essiac dosing. For most of the FACT-B and POMS analyses, we have about 40 Essiac users and between 424 and 447 nonusers available. A power calculation based on independent samples $t$-test demonstrate that one is able to detect a standardized effect size of 0.46 in the standard deviation scale with $80 \%$ power and group sizes of 40 and 424 (the smallest sizes available for any subscale analyses). Thus, we are able to detect a medium effect size even with a relatively small group of users. Although the general lack of significance in the association of Essiac use with the HRQOL and mood states could be attributed to the group size of users, the data nonetheless are indicative of a worsened state of mind in the Essiac group. A larger sample size is unlikely to change any underlying trend in the average differences.

The worse QOL or mood scores in the Essiac users group is perhaps attributable to the fact that this group consists of younger women on an average who are diagnosed at an advanced stage of cancer, a subgroup of patients who are at a worse state of mood or sense of well-being. Choice of Essiac being self-selected, it may be difficult to identify a direct effect of Essiac use, be it positive or negative, on QOL or mood states.

Despite our study's limitations, this trial provides initial information that future studies can build upon. The results of this study suggest several future research directions. For example, animal models could be used to investigate the pharmacokinetics of Essiac and whether higher doses are needed to reach significant concentrations in serum or tar- get tissues. Animal studies could also be used to investigate the effect of Essiac on tumor growth, metastasis, and reoccurrence. Researchers could also use existing large prospective cohorts of women with breast cancer as another way of investigating whether Essiac has an effect on tumor growth, metastasis, and reoccurrence. In addition, a case series in women with breast cancer using Essiac could help to pinpoint why certain women claim to have such dramatic results and why others do not. A case series could also help to more clearly define meaningful outcomes that appear to be changed by Essiac.

\section{CONCLUSIONS}

In conclusion, Essiac does not have a significant effect on HR-QOL or mood states such as anxiety or vigor in women with breast cancer, although Essiac at the doses taken appears to be safe.

\section{ACKNOWLEDGMENTS}

We would like to thank the following students for their help with this project: Amie Litzinger, Laura Shields, Jaklin Iskander, Samantha Ambrozy, and Terri Chan. Natasha Kachan and Julie de Courval provided valuable research support. We would also like to thank Health Canada's Natural Health Products Research Program for funding the TEA-BREAST CANCER Project \# 6814-15-2004/640001.

\section{REFERENCES}

1. Collyar D. Breast cancer: A global perspective. J Clin Oncol 2001;19:101s-105s.

2. Mettlin C. Global breast cancer mortality statistics. CA Cancer J Clin 1999;49:138-144.

3. Canadian Cancer Statistics, 2005. Online document at: www.cancer.ca/vgn/images/portal/cit_86751114/48/28/40159 4768cw_2005stats_en.pdf Accessed November 15, 2005.

4. Radice D, Redaelli A. Breast cancer management: Qualityof-life and cost considerations. Pharmacoeconomics 2003;21: 383-396.

5. Mosconi P, Colozza M, De Laurentiis M, et al. Survival, quality of life and breast cancer. Ann Oncol 2001;12(suppl 3): S15-S19.

6. Roila F, Cortesi E. Quality of life as a primary end point in oncology. Ann Oncol 2001;12(suppl 3):S3-S6.

7. Goodwin P, Black J, Bordeleau L, et al. Health-related aquality-of-life measurement in randomized clinical trials in breast cancer: Taking stock. J Natl Cancer Inst 2003;95:263-281.

8. Boon $\mathrm{H}$. Use of complementary/alternative medicine by breast cancer survivors in Ontario: Prevalence and perceptions. J Clin Oncol 2000;18:2515-2521. 
9. Crocetti E, Crotti N, Feltrin A, et al. The use of complementary therapies by breast cancer patients attending conventional treatment. Eur J Cancer 1998;34:324-328.

10. Jacobson J, Verret W. Complementary and alternative therapy for breast cancer: The evidence so far. Cancer Pract 2001;9: 307-310.

11. Moschen R, Kemmler G, Schweigkofler H, et al. Use of alternative/complementary therapy in breast cancer patients-a psychological perspective. Support Care Cancer 2001;9: 267-274.

12. Rees R, Feigel I, Vickers A, et al. Prevalence of complementary therapy use by women with breast cancer: a populationbased survey. Eur J Cancer 2000;36:1359-1364.

13. Burstein H, Gelber S, Guadagnoli E, et al. Use of alternative medicine by women with early-stage breast cancer. NEJM 1999;340(22):1733-1739.

14. Low Dog T. Traditional and alternative therapies for breast cancer. Altern Ther Health Med 2001;7:36-47.

15. Tamayo C, Richardson M. Presentation at 36th Annual Drug Information Association Meeting. Baltimore, MD, June 17-21, 1999.

16. Eng J. Canadian Cancer Society Information Services: Lessons learned about complementary medicine information needs. Chronic Dis Can 2001;22:3.

17. Kaegi E. Unconventional therapies for cancer: 1. Essiac. Can Med Assoc J 1998;158:897-902.

18. Thomas R. The Essiac report: The True Story of a Canadian Herbal Cancer Remedy and of the Thousands of Lives it Continues to Save, 3rd ed. Los Angeles: Alternative Treatment Information Networks, 1993.

19. Meehan D, Agar J. Doctor's license suspended; some patients still support him. The Grand Rapids Press, April 18, 1997:12.

20. Farragher B. Psychiatric morbidity following the diagnosis and treatment of early breast cancer. Ir J Med Sci 1998;167: 166-169.

21. Brady M. Reliability and validity of the Functional Assessment of Cancer Therapy-Breast Quality of Life instrument. J Clin Oncol 1997;15:974-986.

22. McNair D, Lorr M, Droppleman LF. Manual for the Profile of Mood States. San Diego, CA: Educational and Industrial Testing Services, 1971.

23. Koopman C, Hermanson K, Diamond S, et al. Social support, life stress, pain and emotional adjustment to advanced breast cancer. Psycho-oncology 1998;7:101-111.

24. Zittoun R, Achard S, Ruszniewski M. Assessment of quality of life during intensive chemotherapy or bone marrow transplantation. Psycho-oncology 1999;8:64-73.

25. Breast Cancer, Facts and Figures 2001-2002. Online document at: www.cancer.org/downloads/STT/BrCaFF2001.pdf Accessed June 2005.

26. Box GEP, Cox DR. An analysis of transformations (with discussion). J R Stat Soc B 1964;26:211-252.

27. Cella D, Eaton D, Lai J, et al. Combining Anchor and Distribution-based methods to derive minimal clinincally important differences on the Functional Assessment of Cancer Therapy (FACT) Anemia and Fatigue Scales. J Pain Symptom Manage 2002;24:547-561.

28. Ashikaga T, Bosompra K, O'Brien P, et al. Use of complementary and alternative medicine by breast cancer patients:
Prevalence, patterns and communication with physicians. Support Care Cancer 2002;10:542-548.

29. Lengacher CA, Bennett MP, Kip KE, et al. Frequency of use of complementary and alternative medicine in women with breast cancer. Oncol Nurs Forum 2002;29:1445-1452.

30. Molassiotis A, Scott JA, Kearney N, et al. Complementary and alternative medicine use in breast cancer patients in Europe. Support Care Cancer 2006;14:260-267.

31. Yap KP, McCready DR, Fyles A, et al. Use of alternative therapy in postmenopausal breast cancer patients treated with tamoxifen after surgery. Breast J 2004;10:481-486.

32. Cui Y, Shu X-O, Gao Y, et al. Use of complementary and alternative medicine by Chinese women with breast cancer. Breast Cancer Res Treat 2004;241:1-8.

33. Rakovitch E, Pignol JP, Chartier C, et al. Complementary and alternative medicine use is associated with an increased perception of breast cancer risk and death. Breast Cancer Res Treat 2005;90:139-148.

34. Nagel G, Hoyer H, Katenkamp D. Use of complementary and alternative medicine by patients with breast cancer: Observations from a health-care survey. Support Care Cancer 2004; 12:789-796.

35. Russfield A. Pathology Report, Project No C-144 [Essiac Experiments]. Cambridge, MA: Bio-Research Consultants, Inc., October 8, 1959.

36. Tai J, Cheung S, Wong S, et al. In vitro comparison of Essiac and Flor-Essence on human tumor cell lines. Oncol Rep 2004;11:471-476.

37. U.S. Congress Office of Technology Assistance. Unconventional Cancer Treatments [OTA-H-405]. Washington, DC: U.S. Government Printing Office, 1990:71-75.

38. LeMoine L. Essiac: An historical perspective. Can Oncol Nurse J 1997;7:216-221.

39. Richardson M. Flor-Essence herbal tonic use in North America: A profile of general consumers and cancer patients. HerbalGram 2000;50:40-46.

40. Kaegi E. Essiac: An Information Package. Toronto: CBCRI, 1996.

41. Gray C. Laetrile: Canada's legal position firm but pressure in the South grows. Can Med Assoc J 1997;117:1068-1074.

42. Essiac: An ineffective cancer treatment. Ottawa, Ontario, Canada; Health and Welfare: Canada HaW, Health Protection Branch, February 7, 1989.

43. Shen J, Andersen R, Albert PS, et al. Use of complementary/alternative therapies by women with advanced-stage breast cancer. BMC Complement Altern Med 2002;2:8.

44. Salmenpera L. The use of complementary therapies among breast and prostate cancer patients in Finland. Eur J Cancer Care (Engl) 2002;11:44-50.

Address reprint requests to: Suzanna M. Zick, N.D., M.P.H. University of Michigan Integrative Medicine 715 East Huron Street, Suite $1 W$ Ann Arbor, MI 48104-1555

E-mail: szick@umich.edu 